\title{
Ti:sapphire planar waveguide coherent broadband emitter
}

\author{
T. Bhutta, ${ }^{1}$ R.P.Salathe, ${ }^{2}$ D. P. Shepherd, ${ }^{1}$ R.W. Eason, ${ }^{1}$ and M. Pollnau ${ }^{2}$ \\ 'Optoelectronics Research Centra, University of Southampton, Southampton SO 17 1BJ, United Kingdom \\ ${ }^{2}$ Institute of. Applied Optics, Department of Microtechnique, Swiss Federal Institute of Technology, CH-1015 Lausanne, Switzerland
}

Abstract: We present a broadband ( $130 \mathrm{~nm}$ FWHM around $755 \mathrm{~nm})$ light source for interferometric applications based on a continuous-wave pumped Ti:sapphire planar waveguide. Its brightness is several orders of magnitude higher than from black-body radiation.

\section{Summary}

In recent years, broadband fiber interferometers have become very popular as basic instruments used in optical coherence tomography (OCT) for imaging applications in the biomedical field [1]. A major challenge in the further development and applicability of OCT has been the improvement of both its spatial resolution and dynamic range. The longitudinal resolution is inversely proportional to the optical bandwidth of the light source. Broadband luminescence from transition-metal-ion doped materials (e.g., Ti:sapphire) can significantly improve the longitudinal resolution [2] compared to superluminescent diodes ( $30 \mathrm{~nm}$ FWHM), but the low brightness of its luminescence is insufficient for achieving a useful dynamic range in OCT. Femtosecond lasers have, therefore, been used as large-bandwidth high-brightness light sources, and subcellular imaging has recently been demonstrated in this way [3]. However, current femtosecond light sources do not necessarily meet the requirements of compactness, ease of use, and low cost.

We present here a simple broadband light source based on a Ti:sapphire planar waveguide. It operates in a wavelength region near $800 \mathrm{~nm}$, applicable to the investigation of biotissue and detectable with simple silicon diodes, with a bandwidth comparable to that of a femtosecond light sounce. We generate output powers of several hundreds of $\mu \mathrm{W}$ with high transverse confinement. This level of brightness is several orders of magnitude larger than that obtained from simple broadband light sources based on black-body radiation, whose output power in a single mode is in the sub- $\mu \mathrm{W}$ range [4]. With this brightness, a luminescent light source can provide a dynamic range in OCT that is sufficient to replace a femtosecond light source in a number of applications.

The Ti:sapphire planar waveguide investigated was grown by pulsed laser deposition [5] and contained approximately $0.1 \mathrm{wt} . \%$ of $\mathrm{Ti}_{2} \mathrm{O}_{3}$. The dimensions were $9 \mathrm{~mm}$ in length and approximately $10 \mu \mathrm{m}$ in thickness. The sample was pumped by an all-lines continuous-wave 1-W Ar-ion laser. The pump light was focused into the guiding layer by a $\times 16$ microscope objective. The coupling efficiency was $85 \%$. The effective absorption length was $3.0 \mathrm{~mm}$, leading to $95 \%$ of the launched pump light being absorbed.

Measurements of the output power (Fig. 1) were taken with an incoupling mirror only, which transmitted approximately $86 \%$ of the pump light. The maximum output power was $318 \mu \mathrm{W}$, with a corresponding slope efficiency of $5 \times 10^{-4}$. The spectral bandwidth of the output spectrum (Fig. 2) was $\sim 132 \mathrm{~nm}$ FWHM. While the reflectivity of the incoupling mirror was largest around 730 and $870 \mathrm{~nm}$, the largest increase in spectral output power compared to a measurement without incoupling mirror occurred in the Ti:sapphire gain maximum around $800 \mathrm{~nm}$, indicating that a small gain was present in the one-mirror waveguide configuration. The luminescence mode profile in the unconfined axis was concentrated within an angle similar to that of the diverging single-mode pump beam. In the confined axis, the output was single-mode. Single-mode operation is a requirement for efficient coupling into a single-mode fiber interferometer for OCT. 


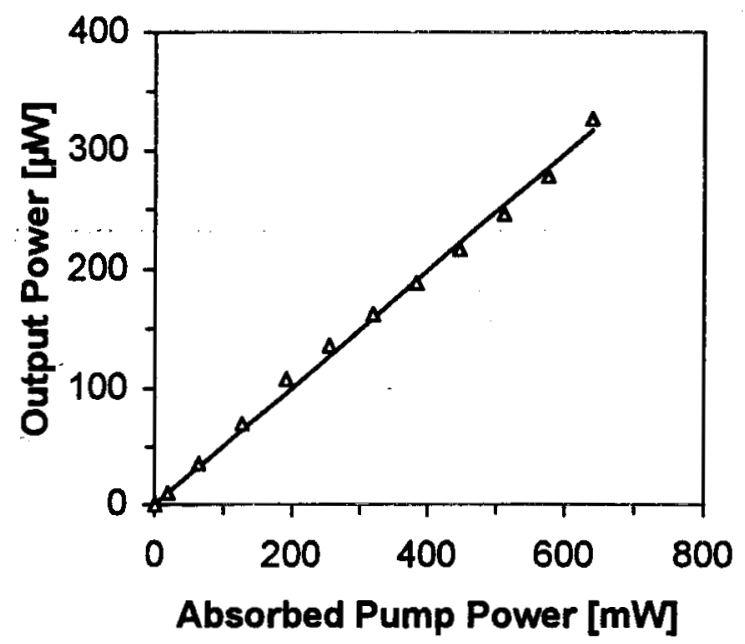

Fig. 1. Input-output ourve of the Ti:sapphire planar waveguide

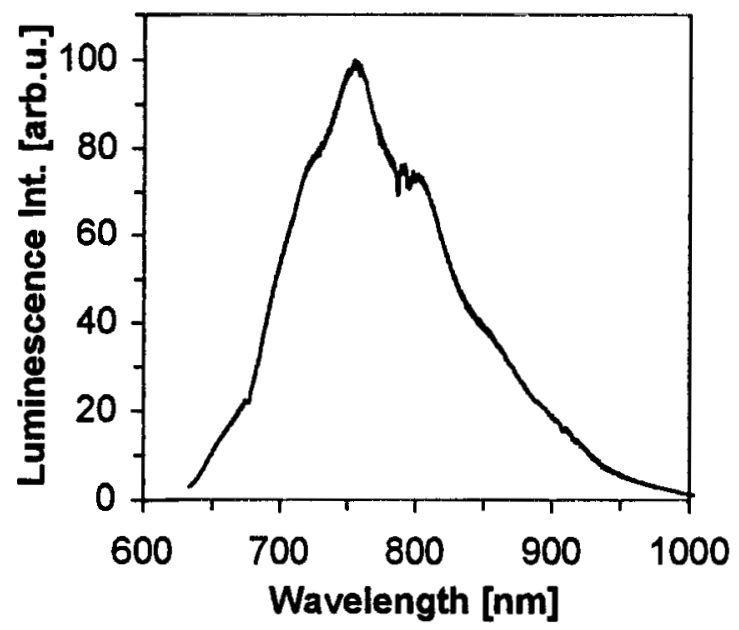

Fig. 2. Luminescence spectrum $(\sim 132 \mathrm{~nm}$ FWHM) of the Ti:sapphire planar waveguide

\section{References}

[1] D. Huang, E. A. Swanson, C. P. Lin, J. S. Schuman, W. G. Stinson, W. Chang, M. R. Hee, T. Flotte, K. Gregory, C. A. Puliafito, and J. G. Fujimoto, "Optical coherence tomography", Science 254, 1178-1181 (1991).

[2] X. Clivaz, F. Marquis-Weible, and R. P. Salathé, "Optical low coherence reflectometry with $1.9 \mu \mathrm{m}$ spatial resolution", Eleotron. Lett. 28, 1553-1555 (1992).

[3] W. Drexler, U. Morgner, F. X. Kärtner, C. Pitris, S. A. Boppart, X. D. Li, E. P. Ippen, and J. G. Fujimoto, "In vivo ultrahigh-resolution optical coherence tomography", Opt. Lett. 24, 1221-1223 (1999).

[4] A. Fercher, C. K. Hitzenberger, M. Sticker, E. Moreno-Barriuso, R. Leitgeb, W. Drexler, and H. Sattmann, "A thermal light souroe technique for optical ooherence tomography", Opt. Commun., in press.

[5] A. A. Anderson, R. W. Eason, M. Jelinek, C. Grivas, D. Lane, K. Rogers, L. M. B. Hickey, and C. Fotakis, "Growth of Ti:sapphire single crystal thin films by pulsed laser deposition", Thin Solid Films 300, 68-71 (1997). 